

\title{
RIESGOS Y CONTROLES EN LOS PROYECTOS DE IMPLEMENTACIÓN DE ERP
}

\author{
Manuel Núñez Eduardo
}

\section{Resumen}

En la actualidad, las empresas trabajan con un alto volumen de información, debido a que cuentan con sistemas que les permite manejar cientos de transacciones al día, como operaciones de venta, compra, producción, planillas, entre otros. Muchas compañías están optando por cambiar sus sistemas de información — desarrollados o adquiridos - por los denominados Enterprise Resource Planning (ERP), implementando los módulos asociados a los procesos de negocio con los cuales laboran. Es necesario tomar en cuenta que todos los procesos de negocio están asociados a riesgos operativos y financieros, los que pueden ser mitigados mediante controles manuales o automáticos. Sin embargo, a pesar de que los ERP manejan un alto nivel de controles automáticos configurables e inherentes, en muchas de sus implementaciones no configuran adecuadamente dichos controles, dejando descubiertos los riesgos mencionados. En este sentido, como antesala a la implementación del ERP, se propone el proyecto de evaluación de riesgos y controles asociados a los procesos como el conjunto de las actividades necesarias para que el nuevo sistema de información (ERP) permita a los procesos de negocio contar con controles fuertes y adecuados para mitigar los riesgos operativos y financieros de la empresa.

Palabras clave: Riesgo operativo / riesgo financiero / control manual / control automático / ERP - Enterprise Resource Planning / interfaz / repositorio de datos. 


\section{La fortaleza de los controles en la implementación de ERP}

Imaginemos que una compañía de retail contrató a una empresa consultora para implementar su Enterprise Resource Planning (ERP), pues quería seguir la tendencia y cambiar su sistema core. ¿De dónde salió esa idea? En una reunión social, donde el gerente financiero del retail escuchó los argumentos del gerente de sistema de otra empresa: "Podrás aumentar el número de transacciones de ventas y el ingreso de efectivo", le comentó.

El lunes siguiente, a primera hora, el equipo del área de sistemas del retail recibía la tarea de investigar acerca de cuál era el ERP que más le convenía. Este ejemplo ficticio se alinea con muchos casos reales, los cuales se enmarcan dentro del boom de implementaciones de ERP para diferentes sectores, como son el retail, la manufactura, las comunicaciones, las empresas periodísticas, la minería, entre otros.

Pero muchas empresas no desarrollan un plan o proyecto para evaluar el ambiente de control donde implementarán el nuevo sistema. Por ejemplo, las compañías jóvenes (de cero a tres años) se están adecuando al uso del ERP adquirido. Es decir, no lo emplean en su máxima potencialidad en el nivel de controles, ya que no conocen todas sus opciones. Otro aspecto recurrente es la resistencia al cambio; esperan realizar sus actividades tal como lo hacían con el sistema anterior, pudiendo encontrar la forma generando una falencia de control. La tercera observación, y quizá la más importante, es que el implementador del ERP suele dejar muchas"puertas abiertas" de seguridad en los procesos de negocio, lo cual puede generar riesgos operativos o financieros. Recordemos que el ERP es altamente configurable y por eso es trascendental considerar en el proyecto de implementación la adecuada configuración de los controles.

Las empresas que cuentan con más de tres años de uso del sistema ERP son más conscientes de los riesgos operativos y financieros y saben qué controles están soportados por el sistema y cuáles faltan implementarse; $y$ entre sus acciones está la de aumentar las funciones de revisión sobre los controles. Esto en lo que se refiere a las empresas reguladas, pero para las que no se encuentran reguladas este paso no asegura en su totalidad el ambiente de control.

Todas estas observaciones son resultado de evaluaciones posteriores, cuando en realidad deberían ser tomadas en cuenta antes de la implementación de un proyecto de ERP. Por ello, se propone un plan de evaluación de riesgos y controles asociados para su validación, antes de efectuar la implementación del ERP, y lograr que la parte operativa del negocio sea eficiente y segura, y mitigue los riesgos asociados. 
La propuesta mencionada es soportada por el modelo GRC, que integra el Gobierno, el Riesgo y el Cumplimiento en la empresa.

De acuerdo con lo indicado, el gobierno define los objetivos del negocio, de donde se desprenden diferentes iniciativas, como el caso del proyecto de implementación de un ERP. Para esto se genera normativas, políticas y procedimientos que rigen las actividades de la empresa para mantener un adecuado gobierno corporativo. Con el fin de que los objetivos del negocio no se distorsionen, surge la necesidad de realizar la evaluación de riesgos sobre el proyecto de implementación del ERP y sobre el sistema en sí. Como respuesta a la identificación de riesgos se llega a la aplicación de controles, cubriendo el aspecto de cumplimiento.

\section{El cambio de los sistemas de información en el mercado empresarial}

Actualmente, las empresas de todos los tamaños soportan sus actividades y procesos por sistemas de información, desde aplicaciones de tipo in-house, compuestas por un repositorio de datos y una máscara o pantalla con la que interactúa el usuario final, hasta sistemas complejos, bien estructurados, que facilitan la gestión de las múltiples operaciones diarias de grandes empresas, llegando a ser adquiridas a proveedores especializados en productos de software para el negocio.

Esta es la realidad peruana en la que se pretende plasmar el presente texto, donde la diversidad de sistemas de información convive con el día a día de la actividad empresarial. Esta diversidad se ha enrumbado por nuevos caminos hacia el actual foco de la industria de software, la estandarización de los sistemas de información core que soportan la columna vertebral de las empresas. Con esta tendencia, se puede apreciar la llegada de los sistemas ERP, que con su alto grado de configuración se adaptan a muchos rubros empresariales, abarcando los sectores retail, minería, manufactura, medios de comunicación, banca y seguros, entre otros.

En el Perú existen varias entidades consultoras, cuya labor es implementar el ERP elegido y comprado por la organización interesada. El objetivo de estas entidades especializadas es dejar el nuevo sistema operando según las definiciones acordadas y evaluadas por el personal competente de la empresa que lo ha adquirido. Es necesario resaltar aquí lo que constituye el punto clave del presente texto: en nuestro país hay proyectos de implementación de ERP que no cuentan con los niveles adecuados para la configuración de los controles automáticos, que permitan mantener un buen balance entre las operaciones de los procesos de negocio y la seguridad de los datos operacionales e información financiera. 


\section{El ERP y su relación con los riesgos en los procesos de negocio y los controles asociados}

Muchas de las empresas, tanto en el ámbito nacional como en el internacional, soportan sus procesos de negocio en sistemas de información. Algunos van desde procesos muy simples hasta niveles complejos. Unos pueden centralizar sus operaciones internamente y en una sola sucursal, mientras que otros pueden tener tercerizados ciertos procesos de negocio y compartir el resto de sus procesos en muchas sucursales o centros. Estos diferentes esquemas de negocios han generado la necesidad, en las empresas, de efectuar desarrollos internos de software, contratar proveedores que desarrollen a medida aplicaciones o adquirir aplicaciones con funcionalidades específicas para cubrir partes de uno o más procesos especiales para el negocio.

A continuación se presenta un cuadro donde se ejemplifica la relación "Complejidad del sistema / Sistema desarrollado-adquirido".

\begin{tabular}{|lll|}
\hline $\begin{array}{c}\text { Categorías de sistemas } \\
\text { de información }\end{array}$ & $\begin{array}{l}\text { Sistemas de menor } \\
\text { complejidad } \\
\text { Cistemas adquiridos }\end{array}$ & \multicolumn{1}{c|}{$\begin{array}{c}\text { Sistemas de mayor } \\
\text { complejidad }\end{array}$} \\
& $\begin{array}{l}\text { Procont } \\
\text { Contasis }\end{array}$ & SAP \\
& & EBS \\
& & Dynamics \\
& Sistema integrado de planificación \\
Sistemas desarrollados & $\begin{array}{l}\text { Gestor de tickets de in- } \\
\text { cidentes y programación de producción }\end{array}$ \\
(in-house) & de aplicaciones & Sistemas Backoffice de operacio- \\
& & nes transaccionales, contables y \\
& & financieras \\
\hline
\end{tabular}

Cuadro 1. Categorías de los sistemas de información y su nivel de complejidad

Elaboración propia.

Todo tipo de desarrollo o de compra de sistema de información tiene como finalidad la automatización y mayor agilidad de una parte, de un proceso o de muchos procesos del negocio. Sin embargo, este objetivo no considera la adecuada evaluación y definición de controles automáticos. Esta cultura se mantiene de forma reactiva; es decir, posterior a tener el (los) proceso(s) automatizado(s), se llega 
a conocer cuáles son los riesgos que no se encuentran cubiertos por controles automáticos del sistema de información, e inclusive no se llega a tener un mapa completo de cuáles son los riesgos presentes de dichos procesos.

Toda organización o empresa tiene riesgos que se encuentran presentes en los procesos de negocio. Algunos riesgos tienen mayor ponderación que otros en un enfoque de criticidad. Es por esto que el riesgo puede ser tratado, de acuerdo con el ISACA (Information System Audit and Control Association) de las siguientes cuatro maneras:

- Mitigado, por medio de aplicación de controles.

- Transferido, a través del traspaso de la operación con riesgo a otro proceso.

- Eliminado, por medio de la eliminación del proceso o subproceso que contenga el riesgo a eliminar.

- Aceptado, declarando la aceptación de la convivencia del riesgo.

De acuerdo con lo mencionado, la mitigación de riesgos incorporando controles automáticos en los aplicativos desarrollados o adquiridos implicaba costes adicionales. Sin embargo, con los sistemas ERP la versatilidad y la flexibilidad de las configuraciones (customizing), permite ampliar la potencialidad en el manejo de controles automáticos en los procesos de negocio, con el fin de mitigar los riesgos asociados.

\section{Riesgos latentes alrededor del proyecto de implementación del ERP}

El ERP es un sistema que permite integrar las operaciones de negocio a través de procesos y cuya columna vertebral es la parte contable financiera. Ahora bien, en estos últimos años se ha ido afianzando la tendencia del cambio de los sistemas legados, los sistemas in-house y otros, por los ERP. Para esto, el mercado peruano cuenta con una amplia gama de socios comerciales (en adelante partner) de las empresas proveedoras del ERP, las cuales se basan normalmente en una metodología establecida por el propio proveedor de dicho sistema para realizar el proyecto de su implementación.

Las empresas adquirientes del ERP (en adelante cliente) efectúan una evaluación de cuál es el que más les conviene, según las experiencias, expectativas, costes, $R O I$, mejor soporte postimplementación, entre otros tópicos importantes, para tener una decisión acertada en la adquisición. Una vez seleccionado el ERP, se decide acerca del partner que implementará el sistema a adquirir. La selección de este es importante en el proyecto de implementación, ya que puede ser que trabaje bajo las buenas prácticas de implementación o que el servicio no satisfaga las definiciones acordadas entre el cliente y el partner, viéndose reflejado en falencias del ERP en el nivel de controles, como producto final. 
Existen casos en los que el partner realiza la implementación tomando estructuras de programas utilizados en trabajos de implementación realizados para otros clientes, a fin de acelerar la fase de desarrollo y configuración del ERP. La consecuencia de esta práctica es que al finalizar el proyecto cabe la probabilidad de que haya programas concurrentes o verticales que no formen parte de las personalizaciones para el cliente y que puedan generar vulnerabilidades en el sistema.

Otro hecho clave en este tipo de proyectos es el adecuado paso de conocimiento del implementador (partner) hacia los responsables funcionales del cliente. Esto es, existen casos en los que el know-how es traspasado durante las pruebas que se realizan durante el proyecto en mención, e incluso hay ocasiones en que el partner no provee de la documentación respectiva de los procesos inmersos en el sistema, lo cual incrementa las dudas que se generan en el cliente en el transcurso de sus operaciones diarias. Esta es la última etapa (la documentación) que debe ser considerada luego de la implementación del ERP por los partners.

Si bien existen muchos otros casos en los que se documenta en detalle el flujo de procesos y controles, y cómo estos se encuentran soportados (configurados) en el ERP, la situación descrita en el párrafo anterior es uno de los factores que puede volver compleja y costosa la evaluación de controles y su correcta adecuación en el ERP para la mitigación de riesgos.

El foco de los proyectos de implementación del ERP está en que la operativa del negocio se alinee con las mejores prácticas del ERP adquirido y, a su vez, que sus procesos de negocio soportados por el nuevo sistema no impacten de manera crítica en las operaciones del día a día. En este sentido, la evaluación por la que pasa el nuevo sistema, luego de la puesta en vivo, es mayoritariamente funcional, dejando de lado el aspecto de los controles automáticos configurables en los procesos de negocio, generando riesgos de índole operativa o financiera.

\section{La conceptualización de los controles de aplicación}

El concepto de controles está soportado por el ISACA, entidad afiliada a la IFAC (International Federation of Accountants).

De acuerdo con el marco de referencia de las mejores prácticas en Tl, el Cobit (Control Objetives for Information and Related Technology), 'control' es definido como "Las políticas, procedimientos, prácticas y estructuras organizacionales diseñadas para proporcionar una garantía razonable de que los objetivos del negocio se alcanzarán, y los eventos no deseados serán prevenidos o detectados." 
Al aplicar el concepto de control a los sistemas de información, por ejemplo a los ERP, podemos usar el término de controles automáticos como la aplicación de las políticas, los procedimientos, las prácticas y las estructuras organizacionales que se encuentran definidas y configuradas en los sistemas de información, y que permiten prevenir la ocurrencia de eventos no deseados en los procesos de negocio automatizados.

\section{Las regulaciones y leyes fortalecen los controles aplicados en los sistemas de información}

En la actualidad, las empresas pasan por diferentes procesos de revisiones, auditorías, consultorías de seguridad de la información, entre muchas otras. Esto se debe a que dichas empresas están reguladas y supervisadas por alguna entidad autónoma del Estado, que vela por los intereses de los de los clientes, como en el caso de la Superintendencia de Banca, Seguros y AFP (SBS), que supervisa los bancos, las compañías de seguros y las AFP garantizando los depósitos de los ahorristas, y los derechos de los asegurados y afiliados, respectivamente. De igual forma sucede con las empresas que desarrollan su actividad bursátil en bolsas extranjeras, las cuales están reguladas por entidades internacionales como la US Securities and Exchange Comission (SEC), la cual, como indica su página web, es "[...] responsable de la implementación de una serie de iniciativas reguladoras requeridas bajo la reforma de Dodd-Frank Wall Street y la actividad de protección al consumidor" (2011, en línea).

Diferente es el caso de las empresas que requieren de informes de las firmas de auditoría o de resultados de los trabajos de consultorías para efectos de presentación al directorio de la empresa o para fines particulares de cada entidad.

Adicionalmente, existen leyes que regulan con mayor detalle los procesos del sistema contable y de las auditorías realizadas a empresas que cotizan en bolsa, como las leyes Sarbaney-Oxley (SOx) e InterCompany Exchange (ICE) (regulación para empresas que cotizan en Bolsa de Australia), que permiten definir claramente el flujo de los procesos de la empresa, detallando las actividades, controles y actores dentro de los flujos, lo que reduce en gran medida los riesgos financieros.

Actualmente, cada uno de los escenarios expuestos en esta sección se encuentran presentes en nuestra realidad peruana. Asimismo, de acuerdo con lo descrito anteriormente, podemos distinguir tres factores principales que permiten a ciertas empresas llegar a tener un mayor nivel de madurez, en lo que respecta a la relación del proyecto de implementación del ERP con adecuados controles manuales y automáticos que mitiguen los riesgos de los procesos de negocio; estos son: 
- Adecuada gestión del riesgo.

- Regulaciones brindadas por entidades externas y del Estado.

- Leyes específicas sobre las actividades de las entidades auditoras y las áreas contable-financieras de la empresa.

Para los tres casos, se mantienen como pilares el adecuado tratamiento de los riesgos, la fortificación del ambiente de control, la definición clara y detallada de los procesos de la empresa y la respectiva tarea fiscalizadora de las actividades que realizan las áreas claves de la empresa regulada.

\section{Convergencia de los riesgos y controles en los procesos de negocio}

A continuación se presenta un gráfico que modela la curva de los procesos óptimos de una empresa (pudiendo ser parte de los dos puntos enumerados anteriormente) y la curva de los mismos procesos sin una adecuada estandarización, modelamiento y control.

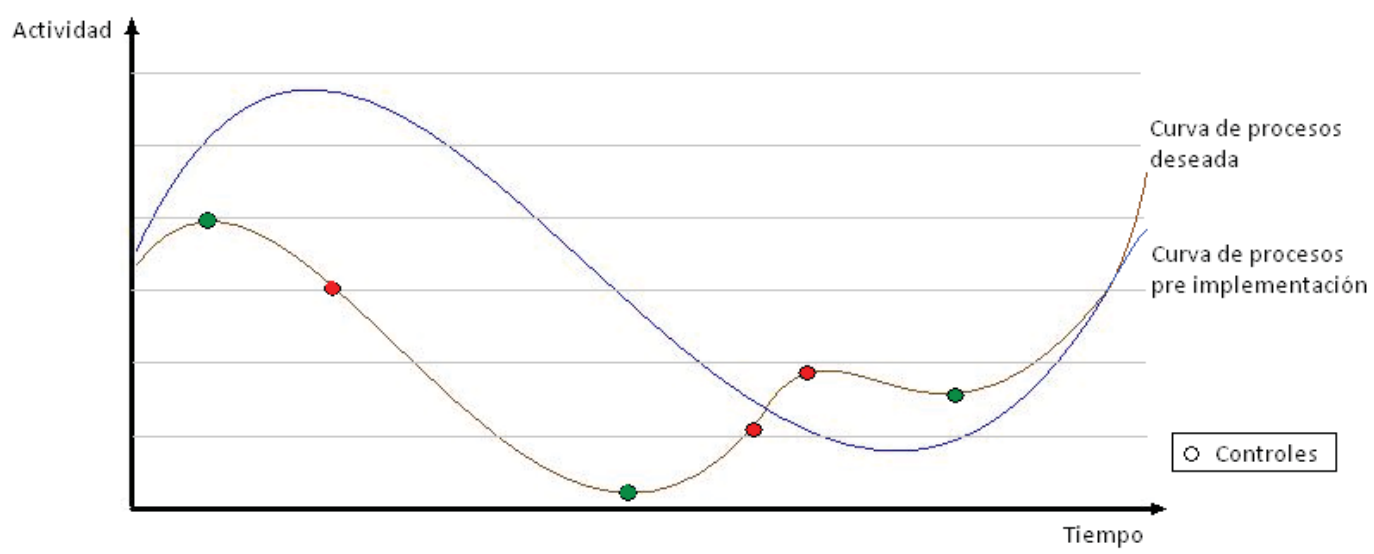

Figura 1. Modelo de variación de las curvas de procesos de negocio

Elaboración propia. 
En la figura 1 se representan dos curvas de diferente color. La curva de azul está basada en la función seno(x), la cual se ha elegido por su simetría, continuidad y curvatura definida para hacer de símil con el proceso deseado, definido, modelado y controlado; mientras que la línea de color marrón representa el proceso que existe en un cliente no regulado o fiscalizado, que aún no pasa por el proyecto de implementación del ERP y mucho menos por el proyecto de evaluación de riesgos y controles asociados.

Adicionalmente, en la misma figura se presenta, a modo de indicadores, los controles que conviven dentro de los procesos del negocio y que permiten mitigar sus riesgos asociados. Algunos de estos se encuentran operando a cabalidad (resaltados de color verde); otros (color rojo) no cubren las eventualidades por las que los riesgos se puedan materializar en el tiempo.

La gráfica representa los casos que normalmente se aprecian en la realidad peruana, en el marco del boom de las implementaciones de los ERP. Estos casos se pueden dar por diferentes factores, desde antes de la concepción del proyecto de implementación, los cuales se mencionarán en los próximos párrafos.

\section{Factores apalancadores de riesgo en los proyectos de implementación de ERP}

Uno de los factores que no se realiza -y que permitiría obtener un producto final (el nuevo sistema ERP instalado) altamente funcional y preparado para manejar los riesgos del negocio - es la concepción y puesta en práctica del proyecto de evaluación de riesgos y controles asociados a los procesos de la empresa, y que está orientado a desarrollar los siguientes objetivos:

a) Evaluar los riesgos identificados por la empresa y que afectan el negocio.

b) Identificar los riesgos que no se hayan sido contemplados inicialmente por la empresa.

c) Evaluar la eficiencia de los controles asociados a los riesgos identificados por la empresa en el primer bullet.

d) Definir nuevos controles como consecuencia de la identificación de nuevos riesgos para evaluar su eficiencia en la mitigación de estos. 
El segundo factor se presenta durante el proyecto de implementación del ERP. En este caso, no es muy regular encontrar una inadecuada selección de estrategia para el traslado de datos (migración de datos) entre el antiguo sistema y el nuevo ERP. Esto se debe a que desde el uso del sistema anterior, los maestros de datos (clientes, materiales, proveedores, precios, empleados) no mantenían las características de validez y exactitud, adoleciendo de redundancia, faltantes e inconsistencias con la realidad, por lo que eran migrados con dichas falencias hacia al ERP. Cuando los procesos de negocio usan estos datos maestros que no han sido corregidos, el resultado del proceso no es el correcto, generando apalancadores a los riesgos del negocio.

En las migraciones actuales de datos se presenta un caso especial. Cuando se pasa de un sistema antiguo a uno nuevo (para nuestros fines a un ERP), los datos más sensibles y significativos que se migran son los "Saldos Contables" de la empresa, lo que unido al hecho del cambio del PCGR (Plan Contable General Revisado) por el PCGE (nuevo Plan Contable General para Empresas, soportado por la ley 28708), incorpora grandes riesgos al proyecto de implementación del ERP. Al tratarse de un caso especial merece ser tratado de manera particular. Sin embargo, cuenta como un factor adicional que se cohesiona al proceso de migración de datos.

El tercer factor, como el anterior, es apreciado durante las fases del proyecto de implementación del ERP e, incluso, puede ser posterior al proyecto. Muchas veces encontramos que los procesos core, los procesos de apoyo y los procesos de control de la empresa (por ejemplo los de ventas, producción, logística, entre otros), están soportados por diferentes sistemas de información (a los cuales denominaremos sistemas satélites), mientras que el proceso contable se encuentra soportado por el ERP.

El escenario descrito genera un esquema híbrido de sistemas de información que deben interactuar entre sí, ya sea por interfaces automáticas, mixtas o manuales. La aplicación de interfaces es crítica y en caso de no identificar todas las interfaces a ser desarrolladas, los datos que deben ser transferidos entre aplicaciones y la frecuencia de la comunicación mutua puede dar como resultado datos incompletos que no reflejan la operativa del negocio.

A continuación se presenta una gráfica que muestra la relación de sistemas satélites con un ERP, que es la base de los procesos de negocio de la empresa. 


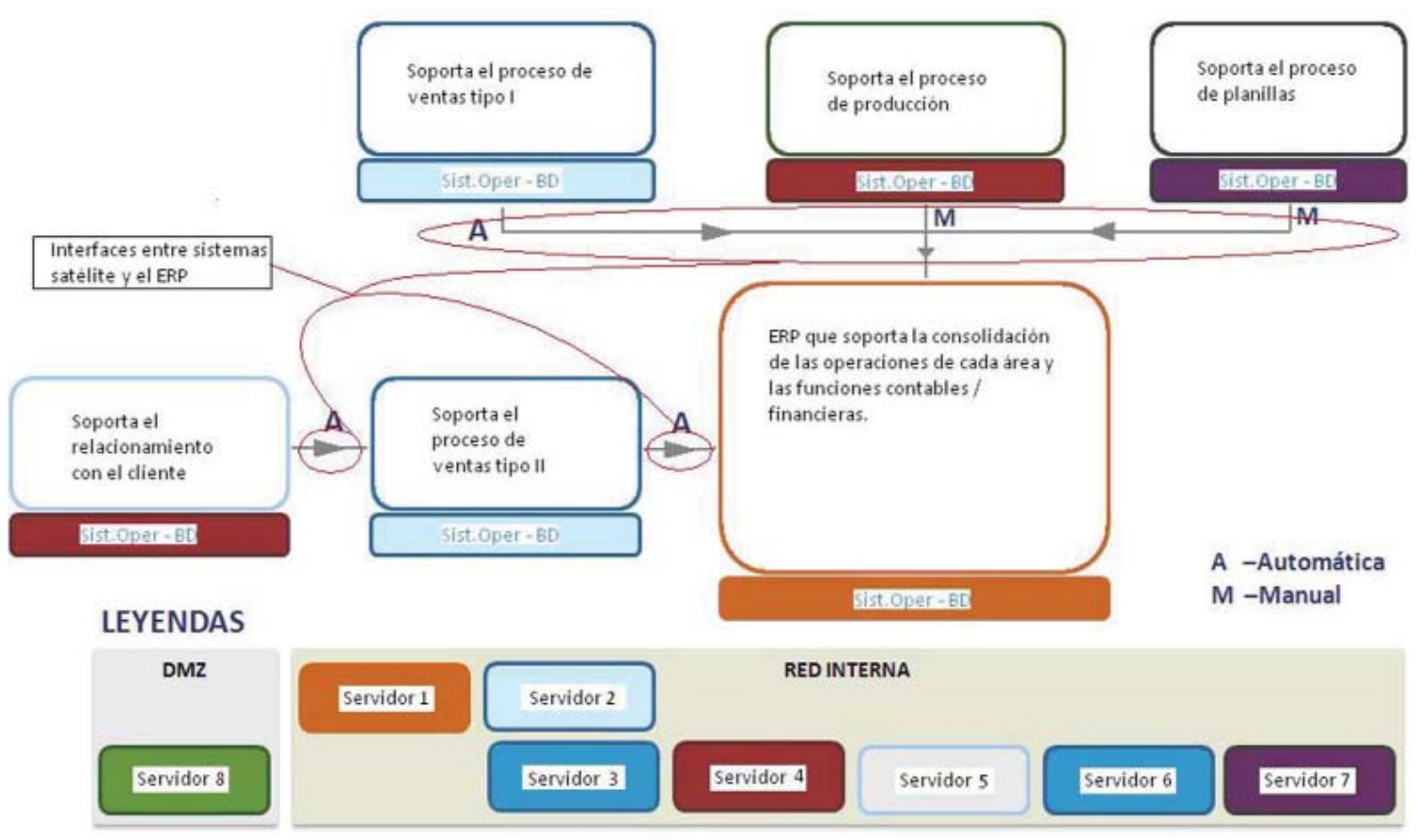

Figura 2. Diagrama relacional de aplicaciones de la empresa

Elaboración propia

En la figura 2 se aprecia que los sistemas de planillas y de producción poseen interfaz manual, mientras que los tres sistemas restantes cuentan con interfaces automáticas hacia el ERP. Asimismo, uno de los temas recurrentes en las empresas que adquieren algún ERP es el hecho de dejar sistemas satélites aislados, sin comunicación de datos (interfaz) con el ERP implementado, hasta después del cierre del proyecto. En este lapso, el riesgo de modificación de datos que no reflejen las operaciones diarias o su falta de integridad es alto, ya que el traspaso de los datos hacia el ERP se hace solo de forma manual o con ayuda del sistema satélite para generar reportes que puedan ser importados al ERP. Para mitigar estos riesgos es necesario que la empresa disponga de controles manuales detectivos bien diseñados y con los objetivos de control claramente definidos. 


\section{Controles: fuente indispensable para mitigar los riesgos del negocio}

El presente artículo ha tenido como objetivo presentar, a grandes rasgos, las situaciones que encontramos actualmente en las empresas que están migrando o que ya cuentan con los denominados ERP. Asimismo, se ha listado algunos de los problemas encontrados en los diferentes sectores y ramos de nuestro mercado empresarial. De igual forma, se ha presentado diversos factores que ayudan a componer los inadecuados controles que incrementan los riesgos de los procesos con que opera la empresa, donde solo algunos están relacionados con las adquisiciones y proyectos de implementación de ERP en el mercado empresarial peruano de la actualidad.

Como corolario de lo desarrollado en las páginas anteriores, se plantea tener presente las siguientes recomendaciones:

- Definir los procedimientos para sostener un adecuado proyecto de implementación del ERP.

- Definir los riesgos asociados a los procesos de negocio y su repercusión en la inclusión del proyecto de implementación de ERP.

- Definir los controles que ayudarán a mitigar los riesgos anteriores.

- Efectuar actividades que aseguren el cumplimiento de los procedimientos y controles.

\section{Glosario de términos}

Concurrente: Desarrollo personalizado para el caso del EBS de Oracle.

Configuración: Adaptar una aplicación software al resto de los elementos del entorno y a las necesidades específicas del usuario.

ERP (Enterprise Resource Planning). Sistema integrado que permite administrar y gestionar las operaciones diarias de la empresa y reflejar su respectiva contabilización para que posteriormente se emitan sus estados financieros.

GRC: Gobierno, riesgo y cumplimiento son los tres términos que describen un amplio rango de actividades dentro de la organización.

Interfaz: Conexión entre diferentes sistemas de información que hace posible la transferencia de datos en línea, por registro manual o por importación manual de archivos generados por el sistema.

In-house: Desarrollo de aplicaciones a medida de las necesidades de la empresa.

Know-how: Conocimientos adquiridos o preexistentes, no siempre de carácter académico, que incluyen técnicas, información secreta, teorías, etcétera. 
Migración de datos: Proceso mediante el cual los datos residentes son traspasados de una base de datos a otra.

Partner: Empresa consultora que brinda servicios o productos, autorizada por el proveedor principal.

Política: Orientaciones o directrices que rigen la actuación de una persona o entidad en un asunto o campo determinado y que se encuentran plasmadas en documentos.

Procedimiento: Relación de métodos que definen cómo ejecutar lo definido por las políticas.

Producto de software: Aplicación desarrollada para usuarios.

Proyecto de implementación: Proyecto que contempla las fases de planificación, análisis, diseño, construcción, estabilización y cierre.

Repositorio de datos: Colección centralizada de data corporativa, histórica y transformada proveniente de sistemas transaccionales.

ROI: Retorno sobre la inversión.

Riesgo: Probabilidad de que una amenaza se convierta en un desastre.

Sistema legado: Sistema de información antiguo pero que continúa siendo utilizado por la organización.

Sistema de información: Conjunto de elementos orientados al tratamiento y administración de datos e información.

Core: Principal y de mayor importancia.

Tercerizado: Proviene del término 'tercerización', el cual se define como la subcontratación (más conocido por el término en inglés outsourcing), que es el proceso económico en el cual una empresa determinada destina a una empresa externa los recursos orientados a cumplir ciertas tareas, por medio de un contrato. Se da sobre todo en el caso de la subcontratación de empresas especializadas.

Vertical: Desarrollo personalizado para el caso del ERP SAP.

\section{Bibliografía}

Commissioner Glassman, Cynthia A. (30 de setiembre del 2002). Sarbanes-Oxley and the idea of "good" governance. http://www.sec.gov/news/speech/spch586. htm [Consulta: 28 de agosto del 2011]. 
IFAC (Federación Internacional de Contadores) (2007). Acerca de IFAC. http://es.ifac. org/about [Consulta: 28 de agosto del 2011].

ISACA (2011). Historia de ISACA. http://www.isaca.org/About-ISACA/History/Pages/ default.aspx [Consulta: 28 de agosto del 2011].

ISACA (16 de agosto del 2010). Standards, guidelines and procedures for information system auditing, pp. 6-328. http://www.isaca.org/Knowledge-Center/ Standards/Documents/ALL-IT-Standards-Guidelines-and-Tools.pdf. [Consulta: 28 de agosto del 2011].

ISACA Journal (2009). "Building Bridges: IT as an Enabler of GRC Convergence". ISACA Journal. Vol. 3. http://www.isaca.org/Journal/Past-Issues/2009/ Volume-3/Pages/Building-Bridges-IT-as-an-Enabler-of-GRC-Convergence. aspx [Consulta: 28 de octubre del 2011].

Ramos Méndez, Gonzalo (20 de octubre del 2011). El plan general contable para empresas, pp. 10-11. http://www.uladech.edu.pe/webuladech/ escuelas/contabilidad/revista/10\%20-\%2011)\%20Gonzalo\%20Ramos\%20 M\%c3\%a9ndez...pdf.

SBS (26 de agosto del 2011). Superintendencia de Banca y Seguros. http://www.sbs. gob.pe/0/modulos/JER/JER_Interna.aspx?ARE=0\&PFL=0\&JER=4

SEC (U.S. Securities and Exchange Commission) (8 de mayo del 2011). http://www. sec.gov/about.shtml [Consulta: 28 de agosto del 2011]. 